\title{
LOD-BOR-FDTD Algorithm for Analyzing Debye Dispersive Media by Bilinear Z Transforms
}

\author{
Pin Zhang \\ Engineering Institute of Corps Engineers, PLA University of Science and Technology \\ Nanjing 210007, China \\ E-mail: pinzhangten@126.com
}

Received: May 16, 2011

Accepted: June 7, 2011

doi:10.5539/mas.v5n4p118

\begin{abstract}
In order to reduce memory usage and improve efficiency, the unconditionally stable locally 1-D (LOD)-FDTD method for bodies of revolution (BOR) is extended to Debye dispersive media based on the bilinear $Z$ transform (BZT) theory. The LOD-BOR-FDTD method is proposed. To validate the Higher efficiency and Lower memory usage of the proposed algorithm, two numerical examples are given. Compared with the 3-D FDTD and ADI-BOR-FDTD result, they show good agreement and at least $80 \%$ of computational time to the ADI counterparts.
\end{abstract}

Keywords: Body of revolution (BOR), Locally 1-D finite difference time-domain method (LOD-FDTD), Rotationally symmetric geometry, Dispersive media

\section{Introduction}

THE dispersive property of bodies of revolution is important for the analysis of wide-band electromagnetic characteristics. The body-of-revolution finite-difference time-domain (BOR-FDTD) method (A. Taflove and S. C. Hagness, 2005)(D. B. Davidson and R. W. Ziolkowski, 1994)(Y. Chen, R. Mittra, and P. Harms, 1996) is an effective means for simulating electromagnetic wave propagation in circularly symmetric structures. Nevertheless, a time step size of the BOR-FDTD is limited by the Courant-Friedrich-Levy (CFL) condition in the conventional FDTD. As a result, the computational time will be increased significantly, when the use of finer space discretization or a large mode number is needed. To conquer the constraint, (H.-L. Chen, B. Chen, Y. Yi, and D.-G. Fang, 2007) presents alternating-direction-implicit(ADI)-BOR-FDTD method. Recently, the locally 1-D (LOD) scheme has been introduced to BOR-FDTD(Jun Shibayama, Bungo Murakami, Junji Yamauchi, and Hisamatsu Nakano, 2009). The LOD method (J. Shibayama, M. Muraki, J. Yamauchi, and H. Nakano, 2005) provides a quite simple algorithm compared with the ADI algorithm. The LOD formulation is a simple type of split-step approach (W. Fu and E. L. Tan, 2004). Although it is first-order accurate in time (J. Shibayama, M. Muraki, R. Takahashi, J. Yamauchi, and H. Nakano, 2006), the numerical results is comparable to the ADI counterparts which has second-order accurate in time. Although the locally 1-D (LOD)-BOR-FDTD is developed, but no dispersive media was considered.

On the other hand, the use of $Z$ transforms (D. M. Sullivan, 1992) to the treatment of dispersive media in FDTD is an attractive alternative, since it has the advantage that the complicated convolution integrals can simply be reduced to algebraic equations, and the relationship between the flux density and the electric field can readily be translated into finite-difference equations. But we found the conventional $Z$ transforms could bring higher error for Dispersive Media. So the bilinear $Z$ transforms $(B Z T)$ is used to avoid error for the trapezoidal integration is more accurate than rectangular integration (D. M. Sullivan, 2000). This fact motivates us to apply the bilinear $Z$ transforms to the LOD-BOR-FDTD for a concise frequency-dependent formulation.

In this letter, we use the BZT method to build an extension of the LOD-BOR-FDTD to Debye dispersive media in order to reduce memory usage and improve efficiency.

\section{Formulation}

Bilinear Z Transforms

For Debye dispersive medium having $p$ poles, the electric flux density $D$ and the electric field $E$ in the frequency domain are related as (A. Taflove and S. C. Hagness, 2005)

$D(\omega)=\varepsilon_{0}\left(\varepsilon_{\infty}+\sum_{p=l}^{N_{p}} \frac{\varepsilon_{s, p}-\varepsilon_{\infty, p}}{1+j \omega \tau_{p}}\right) E(\omega)$

where $\varepsilon_{0}$ is the free-space permittivity, $\varepsilon_{s, p}$ is the static or zero frequency relative permittivity, $\varepsilon_{\infty, p}$ is the relative permittivity at infinite frequency, $\tau_{p}$ is the pole relaxation time, and $N_{p}$ is the number of poles in susceptibility response. 
Taking bilinear $Z$ transforms of(1)by $\quad 1 / j \omega \rightarrow\left(1+z^{-1}\right) / 2\left(1-z^{-1}\right), \quad 1 /(\alpha+j \omega) \rightarrow\left(1+z^{-1} e^{-\alpha \Delta t}\right) / 2\left(1-z^{-1} e^{-\alpha \Delta t}\right)$ and $D=\varepsilon_{0} \varepsilon_{r}(z) E(z) \Delta t$ in which the normalised expression of field components (A. Taflove and S. C. Hagness, 2005) is used yields the following equation in the $Z$ domain:

$$
D(z)=\varepsilon_{0} \varepsilon_{\infty} E(z)+\sum_{p=1}^{N_{p}} S(z)
$$

Where

$$
\begin{aligned}
S(z) & =\frac{\Delta t \varepsilon_{0}\left(1+z^{-1} e^{-\Delta t / \tau_{p}}\right)\left(\varepsilon_{s, p}-\varepsilon_{\infty, p}\right) / \tau_{p}}{2\left(1-z^{-1} e^{-\Delta t / \tau_{p}}\right)} E(z) \\
& =e^{-\Delta t / \tau_{p}} z^{-1} S(z)+\frac{\Delta t \varepsilon_{0}\left(\varepsilon_{s, p}-\varepsilon_{\infty, p}\right)\left(1+z^{-1} e^{-\Delta t / \tau_{p}}\right)}{2 \tau} E(z)
\end{aligned}
$$

in which $\Delta t$ and $z^{-l}$ represent the time period and the delay of $l$ time periods, respectively. We readily translate (2), (3) into the finite difference equations in the time domain as

$$
\begin{aligned}
& E^{n}=\frac{D^{n}-S^{n-l} \sum_{p=1}^{N} e^{-\Delta t / \tau_{p}}-\sum_{p=1}^{N} \frac{\Delta t \varepsilon_{0}}{2 \tau_{p}}\left(\varepsilon_{s, p}-\varepsilon_{\infty, p}\right) e^{-\Delta t / \tau_{p}} E^{n-1}}{\varepsilon_{0} \varepsilon_{\infty}+\sum_{p=1}^{N} \frac{\Delta t \varepsilon_{0}}{2 \tau_{p}}\left(\varepsilon_{s, p}-\varepsilon_{\infty, p}\right)} \\
& S^{n}=e^{-\Delta t / \tau_{p}} S^{n-1}+\frac{\Delta t \varepsilon_{0}\left(\varepsilon_{s, p}-\varepsilon_{\infty, p}\right)}{2 \tau} E^{n}+\frac{\Delta t \varepsilon_{0}\left(\varepsilon_{s, p}-\varepsilon_{\infty, p}\right) e^{-\Delta t / \tau_{p}}}{2 \tau} E^{n-1}
\end{aligned}
$$

Combined Debye media with LOD-BOR-FDTD

We now solve (4),(5) combined with the following standard LOD-BOR-FDTD equations with the field normalization (Jun Shibayama, Bungo Murakami, Junji Yamauchi, and Hisamatsu Nakano, 2009). For simplicity, we will examine the case for mode number is 0 :

$$
\begin{aligned}
& E_{\rho}^{n+1 / 2}=E_{\rho}^{n}(6 \mathrm{a}) \quad \frac{E_{\phi}^{n+1 / 2}-E_{\phi}^{n}}{\Delta t / 2}=\frac{1}{\varepsilon}\left(\frac{\partial H_{\rho}^{n+1 / 2}}{\partial z}+\frac{\partial H_{\rho}^{n}}{\partial z}\right) \\
& \frac{E_{z}^{n+1 / 2}-E_{z}^{n}}{\Delta t / 2}=\frac{1}{\varepsilon \rho}\left(\frac{\partial\left(\rho H_{\phi}^{n+1 / 2}\right)}{\partial \rho}+\frac{\partial\left(\rho H_{\phi}^{n}\right)}{\partial \rho}\right) \\
& \frac{H_{\rho}^{n+1 / 2}-H_{\rho}^{n}}{\Delta t / 2}=\frac{1}{\mu}\left(\frac{\partial E_{\phi}^{n+1 / 2}}{\partial z}+\frac{\partial E_{\phi}^{n}}{\partial z}\right) \\
& \frac{H_{\phi}^{n+1 / 2}-H_{\phi}^{n}}{\Delta t / 2}=\frac{1}{\mu}\left(\frac{\partial E_{Z}^{n+1 / 2}}{\partial \rho}+\frac{\partial E_{Z}^{n}}{\partial \rho}\right) \quad(6 \mathrm{e}) \quad H_{z}^{n+1 / 2}=H_{z}^{n}
\end{aligned}
$$

for the first step and

$$
\begin{aligned}
& E_{\rho}^{n+1}+\frac{\Delta t}{2 \varepsilon} \frac{\partial H_{\phi}^{n+1}}{\partial z}=E_{\rho}^{n+1 / 2}-\frac{\Delta t}{2 \varepsilon} \frac{\partial H_{\phi}^{n+1 / 2}}{\partial z} \\
& E_{\phi}^{n+1}+\frac{\Delta t}{2 \varepsilon} \frac{\partial H_{z}^{n+1}}{\partial \rho}=E_{\phi}^{n+1 / 2}-\frac{\Delta t}{2 \varepsilon} \frac{\partial H_{z}^{n+1 / 2}}{\partial \rho} \\
& E_{z}^{n+1}=E_{z}^{n+1 / 2}(7 \mathrm{c}) \\
& H_{\phi}^{n+1}+\frac{\Delta t}{2 \mu} \frac{\partial E_{\rho}^{n+1}}{\partial z}=H_{\phi}^{n+1 / 2}-\frac{\Delta t}{2 \mu} \frac{\partial E_{\rho}^{n+1 / 2}}{\partial z} \\
& H_{z}^{n+1}+\frac{\Delta t}{2 \mu \rho} \frac{\partial\left(\rho E_{\phi}^{n+1}\right)}{\partial \rho}=H_{z}^{n+1 / 2}-\frac{\Delta t}{2 \mu \rho} \frac{\partial\left(\rho E_{\phi}^{n+1 / 2}\right)}{\partial \rho}
\end{aligned}
$$

for the second step.

We now calculate the $E$ and $H$ from $n$ time steps to $n+1$.

1) Substituting (7c) into (6c)(6e) equations, we get

$$
\begin{aligned}
& \frac{D_{z}^{n+1}-D_{z}^{n}}{\Delta t / 2}=\frac{1}{\varepsilon \rho}\left[\frac{\partial\left(\rho H_{\phi}^{n+1 / 2}\right)}{\partial \rho}+\frac{\partial\left(\rho H_{\phi}^{n}\right)}{\partial \rho}\right] \\
& \frac{H_{\phi}^{n+1 / 2}-H_{\phi}^{n}}{\Delta t / 2}=\frac{1}{\mu}\left(\frac{\partial E_{z}^{n+1}}{\partial \rho}+\frac{\partial E_{z}^{n}}{\partial \rho}\right)
\end{aligned}
$$

Substituting (4), $\left(6 \mathrm{e}^{*}\right)$ into $\left(6 \mathrm{c}^{*}\right)$, and implicitly solve the resultant tridiagonal equation, we get $E_{z}^{n+1}$. And then 
explicitly solve (6e*), (4) and (5), getting $D_{z}^{n+1}, H_{\phi}^{n+1 / 2}, S_{z}^{n+1}$.

2) Substituting (7d) into (6b) (6d) equations, we get

$$
\begin{aligned}
& \frac{H_{\rho}^{n+1}-H_{\rho}^{n}}{\Delta t / 2}=\frac{1}{\mu}\left(\frac{\partial E_{\phi}^{n+1 / 2}}{\partial z}+\frac{\partial E_{\phi}^{n}}{\partial z}\right) \\
& \frac{E_{\phi}^{n+1 / 2}-E_{\phi}^{n}}{\Delta t / 2}=\frac{1}{\varepsilon}\left(\frac{\partial H_{\rho}^{n+1}}{\partial z}+\frac{\partial H_{\rho}^{n}}{\partial z}\right)
\end{aligned}
$$

Substituting $\left(6 \mathrm{~b}^{*}\right)$ into $\left(6 \mathrm{~d}^{*}\right)$, we get $H_{\rho}^{n+1}$. And then explicitly solve $\left(6 \mathrm{~b}^{*}\right)$, getting $E_{\phi}^{n+1 / 2}$.

3) Substituting (6a) into (7a)(7e) equations, we get

$$
\begin{aligned}
& \frac{D_{\rho}^{n+1}-D_{\rho}^{n}}{\Delta t / 2}=-\frac{1}{\varepsilon}\left(\frac{\partial H_{\phi}^{n+1}}{\partial z}+\frac{\partial H_{\phi}^{n}}{\partial z}\right) \\
& \frac{H_{\phi}^{n+1}-H_{\phi}^{n+1 / 2}}{\Delta t / 2}=-\frac{1}{\mu}\left(\frac{\partial E_{\rho}^{n+1}}{\partial z}+\frac{\partial E_{\rho}^{n}}{\partial z}\right)
\end{aligned}
$$

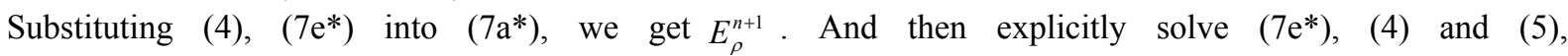
getting $D_{\rho}^{n+1}, H_{\phi}^{n+1}, S_{\rho}^{n+1}$.

4) Substituting (6f) into (7f) (7b) equations, we get

$$
\begin{aligned}
& \frac{H_{z}^{n+1}-H_{z}^{n}}{\Delta t / 2}=-\frac{1}{\mu \rho}\left[\frac{\partial\left(\rho E_{\phi}^{n+1}\right)}{\partial \rho}+\frac{\partial\left(\rho E_{\phi}^{n+1 / 2}\right)}{\partial \rho}\right] \\
& \frac{E_{\phi}^{n+1}-E_{\phi}^{n+1 / 2}}{\Delta t / 2}=-\frac{1}{\varepsilon}\left(\frac{\partial H_{z}^{n+1}}{\partial \rho}+\frac{\partial H_{z}^{n}}{\partial \rho}\right)
\end{aligned}
$$

Substituting (7b*) into (7f*) equations, we get $H_{z}^{n+1}, E_{\phi}^{n+1}$. Then, the $E$ and $H$ are updated from $n$ to $n+1$.

\section{Numerical Results}

To verify the proposed algorithm and accuracy, two numerical examples we analysed are shown. In all cases, the $\theta$-polarized plane wave is introduced by defining a set of equivalent currents on a closed Huygen's surface (D. E. Merewether, R. Fisher, and F. W. Smith, 1980). The Computational domains are truncated by PML cells which have $n=8$ layers (Jun Shibayama, Bungo Murakami, Junji Yamauchi, and Hisamatsu Nakano, 2009).

As a first example (H.-L. Chen, B. Chen, Y. Yi, D.-G. Fang and Heng Liu, 2009), a typical first order Debye material is water with the parameters $\varepsilon_{s}=80, \varepsilon_{\infty}=5.27, \tau=1.0 \times 10^{-11}$. Here, the scattering from a water sphere with radius of $420 \mu \mathrm{m}$ is calculated. The grid size is $\Delta \rho=\Delta z=10 \mu \mathrm{m}$ and mode number ranges from 0 to 6 . Fig.1(a) shows the back scattered radar cross section (RCS) for different CFLN values, which is defined as $C F L N=\Delta t / \Delta t_{F D T D}$, where $\Delta t_{F D T D}$ is the maximum FDTD time step. For the purpose of comparison, the result obtained from three-dimensional (3-D) FDTD method with grid size $\Delta x=\Delta y=\Delta z=10 \mu m$ is also given. Fig. 1(b) shows the errors of the LOD versus ADI method. Compared with the theoretical value, they show good agreement. The errors in time domain are also tabulated, which are evaluated using the following expression:

$$
\operatorname{Error}(\%)=100 \times\left\|E_{\text {far }}-E_{\text {far }}^{\text {ref }}\right\| /\left\|E_{\text {far }}^{\text {ref }}\right\|
$$

where $E_{\text {far }}^{\text {ref }}$ is the far field in time domain calculated using 3-D FDTD, $\|\bullet\|$ represents the Euclid norm operation.

The second example, we calculate the back scattered RCS of a cold plasma cylinder with height of $6 \mathrm{~cm}$ and radius of $3 \mathrm{~cm}$ illuminated by perpendicularly incident plane wave. The complex relative permittivity of the plasma medium is defined as

$\varepsilon_{r}=1+\omega_{p}^{2} /\left(j \omega V_{c}-\omega^{2}\right)$

where $\omega_{p}$ is the radian plasma frequency and $V_{c}$ is the collision

frequency. It has been shown that (K. S. Kunz and R. J. Luebbers, 1993), with the transformation $\mathcal{E}_{r}=1+x_{d}+\sigma /\left(j \varepsilon_{0} \omega\right)$, the plasma medium behaves like a Debye medium with a conductivity, and a negative susceptibility $x_{d}=-\omega_{p}^{2} / V_{c}^{2} /\left(1+j \omega / V_{c}\right)$. The grid size is $\Delta \rho=\Delta z=0.5 \mathrm{~mm}$ and mode number ranges from 0 to 9. The plasma considered has a plasma frequency $\omega_{p}$ of $4.0 \times 10^{10} \mathrm{rad} / \mathrm{s}$ and a collision frequency $V_{c}$ of $20 \mathrm{GHz}$. Again, we compare the results of the proposed method with that of the conventional 3-D FDTD method with grid size $\Delta x=\Delta y=\Delta z=0.5 \mathrm{~mm}$. As shown in Fig. 2(a), good agreements are achieved even 
if the time step of the ADI-BOR-FDTD is four times that used in the conventional FDTD method. Fig. 2(b) shows the errors of the LOD versus ADI method. Good agreement is expressed.

\section{Conclusion}

In this letter, the LOD-BOR-FDTD method has been extended to solve electromagnetic problems in Debye dispersive media using the bilinear $Z$ transforms. The LOD-BOR-FDTD combine with the BZT offers quite simple algorithm with a subsequent reduction in the computational time, maintaining numerical results identical to the ADI counterparts. Numerical results indicate that the presented method is valid and efficient. Compared with the 3-D FDTD and ADI-BOR-FDTD results, they show good agreement and at least $45 \%$ and $80 \%$ of running time, respectively. For open region problems, efficient absorbing boundary conditions such as perfectly matched layers (I. Ahmed, E. Li, K. Krohne, 2007)(V. E. do Nascimento, B. H. V. Borges, and F. L. Teixeira, 2006) for LOD-BOR-FDTD are required. The proposed method has reduced memory usage and improved efficiency.

\section{References}

A. Taflove and S. C. Hagness. (2005). Computational Electrodynamics: The Finite-Difference Time-Domain Method, 3rd ed. Norwood, MA: Artech House, 2005, ch. 12.

D. B. Davidson and R. W. Ziolkowski. (1994). Body-of-revolution finite-difference time-domain modeling of space-time focusing by a three-dimensional lens, J. Opt. Soc. Amer. A, vol. 11, no. 4, pp. 1471-1490, Apr. 1994.

D. E. Merewether, R. Fisher, and F. W. Smith. (1980). On implementing a numeric Huygen's source scheme in a finite difference program to illuminate scattering bodies, IEEE Trans Nucl. Sci, vol. NS-27, no. 6, pp. 1829-1833, Dec. 1980.

D. M. Sullivan. (1992). Frequency-dependent FDTD methods using $\mathrm{Z}$ transforms, IEEE Trans. Antennas Propag., vol. 40, no. 10, pp. 1223-1230, Oct. 1992.

D. M. Sullivan. (2000). Electromagnetic simulation using the FDTD method, IEEE Press Seres on RF and Micro Tech., 2000.

H.-L. Chen, B. Chen, Y. Yi, and D.-G. Fang. (2007). Unconditionally stable ADI-BOR-FDTD algorithm for the analysis of rotationally symmetric geometries, IEEE Microw. Wireless Compon. Lett., vol. 17, no. 4, pp. 304-306, Apr. 2007.

H.-L. Chen, B. Chen, Y. Yi, D.-G. Fang and Heng Liu. (2009). Extension of the ADI-BOR-FDTD Method to Debye Dispersive Media, IEEE Microw. Wireless Compon. Lett., vol. 19, no. 6, pp. 344-346, Jun. 2009.

I. Ahmed, E. Li, K. Krohne. (2007). Convolutional Perfectly Matched Layer for an Unconditionally Stable LOD-FDTD Method, IEEE Microwave and Wireless Components Letters, vol. 17, no. 12, pp. 816-819, Dec. 2007.

J. Shibayama, M. Muraki, J. Yamauchi, and H. Nakano. (2005). Efficient implicit FDTD algorithm based on locally one-dimensional scheme, Electron. Lett., vol. 41, no. 19, pp. 1046-1047, Sep. 2005.

J. Shibayama, M. Muraki, R. Takahashi, J. Yamauchi, and H. Nakano. (2006). Performance evaluation of several implicit FDTD methods for optical waveguide analyses, J. Lightw. Technol., vol. 24, no. 6, pp. 2465-2472, Jun. 2006.

Jun Shibayama, Bungo Murakami, Junji Yamauchi, and Hisamatsu Nakano. (2009). LOD-BOR-FDTD Algorithm for Efficient Analysis of Circularly Symmetric Structures, IEEE Microw.Wireless compon. Lett., vol 19 no.2, pp.56-58, Feb 2009.

K. S. Kunz and R. J. Luebbers. (1993). The Finite Difference Time Domain Method for Electromagnetics. Boca Raton, FL: CRC Press, 1993.

V. E. do Nascimento, B. H. V. Borges, and F. L. Teixeira. (2006). Splitfield PML implementations for the unconditionally stable LOD-FDTD method, IEEE Microw. Wireless Compon. Lett., vol. 16, no. 7, pp. 398-400, Jul. 2006.

W. Fu and E. L. Tan. (2004). Development of split-step FDTD method with higher order spatial accuracy, Electron. Lett., vol. 40, no. 20, pp. 1252-1254, Sep. 2004.

Y. Chen, R. Mittra, and P. Harms. (1996). Finite-difference time-domain algorithm for solving Maxwell's equations in rotationally symmetric geometries, IEEE Trans. Microw. Theory Tech., vol. 44, no. 6, pp. 832-839, Jun. 1996. 


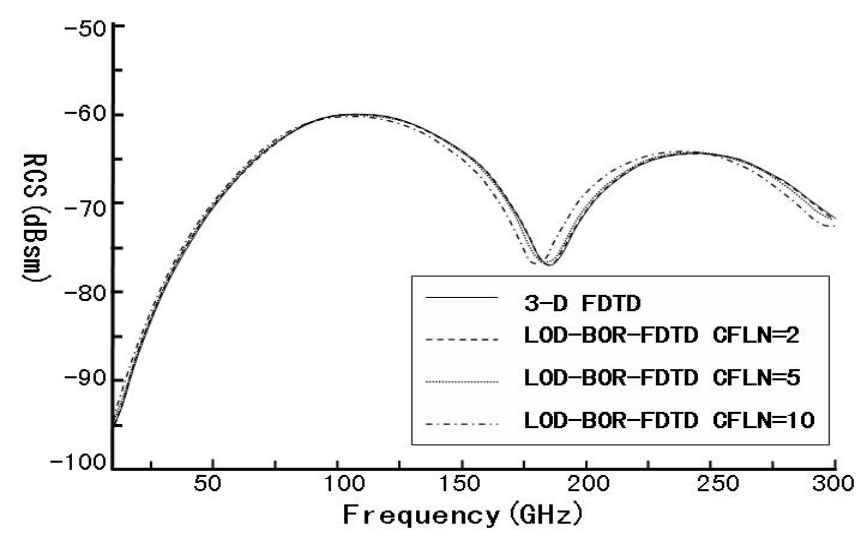

(a)

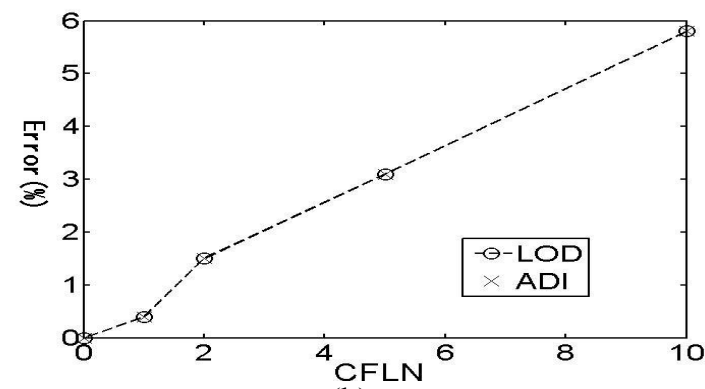

(b)

Figure 1. Backscattering from the water sphere is showed in(a) and errors of the LOD versus ADI method is showed in(b).

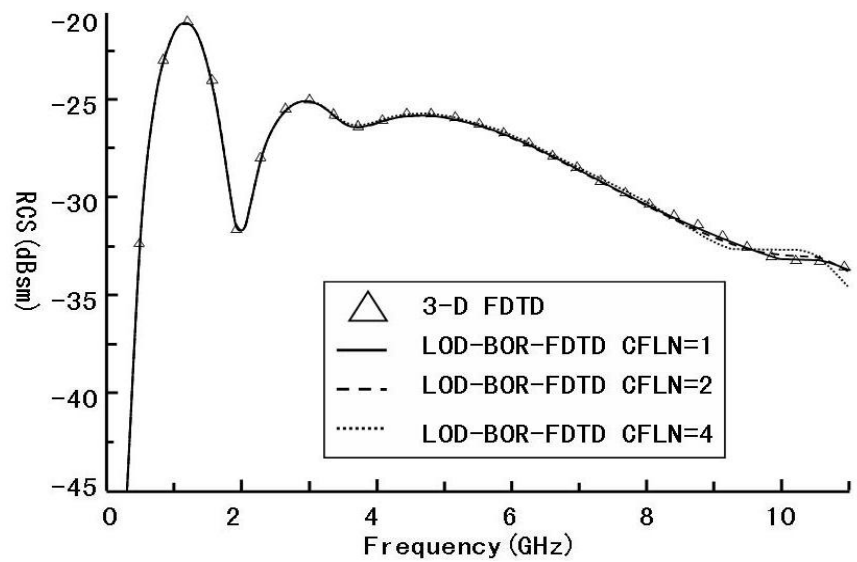

(a)

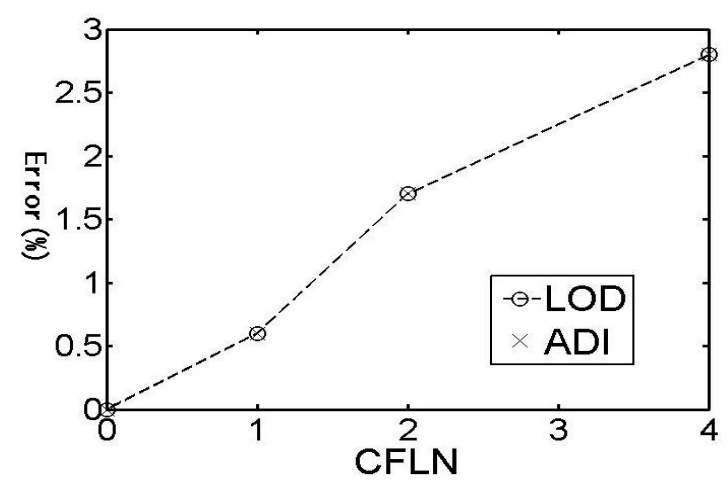

(b)

Figure 2. Back scattered RCS of the plasma cylinder is showed in(a) and errors of the LOD versus ADI method is showed in(b). 